\title{
BURNOUT E COPING EM ATLETAS DE VOLEIBOL: UMA ANÁLISE LONGITUDINAL
}

BURNOUT AND COPING AMONG VOLLEYBALL ATHLETES: A LONGITUDINAL ANALYSIS

AGOTAMIENTO Y AFRONTAMIENTO EN ATLETAS DE VOLEIBOL: UN ANÁLISIS LONGITUDINAL

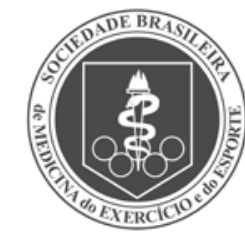

Artigo Original

Original ARTICLE

Artículo Original
Daniel Alvarez Pires ${ }^{1}$

(Educador Físico)

Maurício Gattás Bara Filho²

(Educador Físico)

Paula Barreiros Debien²

(Educadora Física)

Danilo Reis Coimbra ${ }^{3}$

(Educador Físico)

Herbert Ugrinowitsch ${ }^{4}$

(Educador Físico)

1. Universidade Federal do Pará (UFPA) - Campus Universitário de Castanhal, Educação Física, Castanhal, PA, Brasil.

2. Universidade Federal de Juiz de Fora (UFJF), Educação Física, Juiz de Fora, MG, Brasil.

3. Universidade do Estado de Santa Catarina (UDESC), Educação Física,

Florianópolis, SC, Brasil.

4. Universidade Federal de Minas Gerais (UFMG), Educação Física,

Belo Horizonte, MG, Brasil.

\section{Correspondência:}

Universidade Federal do Pará. Campus Universitário de Castanhal. Avenida dos Universitários, s/n, Jaderlândia, Castanhal, PA, Brasil. 68746-360.danielpires@ufpa.br

\section{RESUMO}

Introdução: No contexto esportivo, uma das principais recomendações para o melhor entendimento sobre a síndrome de burnout e o coping é a realização de estudos longitudinais que possam descrever o processo de desenvolvimento da síndrome e as estratégias empregadas para o seu enfrentamento. Objetivos: Analisar as percepções das dimensões de burnout e as estratégias de coping durante uma temporada esportiva, e correlacionar as características temporais de burnout e das estratégias de coping. Métodos: Mediante delineamento longitudinal, foram acompanhados 15 atletas profissionais de voleibol do sexo masculino (média de idade $=24,00 \pm 3,55$ anos) participantes da Superliga Nacional. Para mensurar as dimensões de burnout e as estratégias de coping foram utilizadas as versões traduzidas do Athlete Burnout Questionnaire e do Athletic Coping Skills Inventory. A coleta de dados foi realizada em quatro momentos de uma temporada esportiva. O comportamento dos indicadores de burnout e coping foi verificado através do teste de Friedman, com post hoc de Wilcoxon, enquanto que o coeficiente de correlação de Spearman (rho) foi empregado para verificar as correlações entre os indicadores de burnout e de coping. Resultados: Foi observado aumento significativo da desvalorização esportiva e do índice global de burnout com o transcorrer da temporada, porém não foram encontradas diferenças significativas no emprego das estratégias de coping nos quatro momentos. Foram observadas correlações inversas e significativas entre a dimensão de burnout, senso reduzido de realização esportiva e a estratégia de coping, confiança/motivação, em todos os momentos da temporada. Conclusão: Os achados evidenciaram aumento na percepção de burnout nos atletas a partir do acúmulo de treinamentos e competições, bem como da exigência por ótimo desempenho na competição-chave. O estudo também apontou a relevância do fator motivacional no surgimento do burnout.

Descritores: esgotamento profissional; adaptação psicológica; voleibol.

\section{ABSTRACT}

Introduction: In the context of sports, one of the main recommendations for a better understanding of burnout syndrome and coping is to carry out longitudinal studies that describe the process of development of the syndrome, and the strategies used for its prevention and control. Objectives: To analyze perceptions of the dimensions of burnout and coping strategies during a sporting season, and to correlate the temporal characteristics of burnout and coping strategies. Methods: Through a longitudinal design, the study included 15 male volleyball professional athletes (aged $24.00 \pm 3.55$ years) participating in the Superliga Nacional (Brazilian Volleyball Super League). Each participant completed the translated versions of the Athlete Burnout Questionnaire and Athletic Coping Skills Inventory. Data were collected in four stages of a sporting season. The behavior of the burnout and coping indicators were verified by the Friedman test, with the Wilcoxon post hoc test, and Spearman's correlation coefficient (rho) was used to verify the correlations between the burnout and coping indicators. Results: A significant increase was observed in sport devaluation and overall levels of burnout as the season progressed, but there were no significant differences in the use of coping strategies at all four times. Significant inverse correlations were observed between the dimension burnout, reduced sense of sport accomplishment, and the coping strategy, confidence/motivation at all four times of the season. Conclusion: The results show an increase in the perception of burnout in the athletes with the build-up of training and competition, as well as the requirement for optimal performance in the main competition. The study also showed the importance of the motivational factor in the emergence of burnout.

Keywords: burnout, professional; adaptation, psychological; volleyball.

\section{RESUMEN}

Introducción: En el contexto deportivo, una de las principales recomendaciones para una mejor comprensión del sindrome de burnout y afrontamiento es realizar estudios longitudinales para describir el proceso de desarrollo del sindrome y las estrategias empleadas para afrontamiento. Objetivos: Analizar la percepción de dimensiones del burnout y estrategias de afrontamiento durante una temporada deportiva, y correlacionar las características temporales de burnout y estrategias de afrontamiento. Métodos: Mediante diseño longitudinal, se siguieron 15 atletas profesionales de voleibol masculino (edad media $=24,00 \pm 3,55$ años) que participan en la Superliga Nacional Brasileña. Para identificar las dimensiones de burnout y afrontamiento se utilizaron las versiones traducidas del Athlete Burnout Questionnaire y del Athletic Coping Skills Inventory. La recolección de datos se llevó a cabo en 
cuatro etapas de una temporada deportiva. El comportamiento de los indicadores de burnout y de afrontamiento se verificó mediante la prueba de Friedman, con post hoc de Wilcoxon, mientras que se utilizó el coeficiente de correlación de Spearman (rho) para verificar las correlaciones entre los indicadores de burnout y afrontamiento. Resultados: Se observó un aumento significativo de la devaluación del deporte y el nivel general del burnout con el transcurso de la temporada, pero no hubo diferencias significativas en el uso de estrategias de afrontamiento en los cuatro momentos. Se observaron correlaciones inversas significativas entre la dimensión del burnout, sensación reducida de logro deportivo y la estrategia de afrontamiento, confianza/motivación, en todos los momentos de la temporada. Conclusión: Los resultados muestran un aumento en la percepción de burnout en deportistas debido a la acumulación de entrenamiento y competiciones, así como el requisito para un rendimiento óptimo en la competición clave. El estudio también señaló la importancia del factor de motivación en la aparición del burnout.

Descriptores: agotamiento profesional; adaptación psicológica; voleibol.

\section{INTRODUÇÃO}

A síndrome de burnout é considerada uma reação ao estresse crônico e afeta profissionais de diferentes áreas. Recentemente, o burnout passou a ser investigado também no ambiente esportivo ${ }^{1}$. No caso de atletas, o burnout se origina na incompatibilidade de planos e metas iniciais com as demandas crônicas de cunhos sociais, psicológicas e fisiológicas da modalidade esportiva². Esta incompatibilidade pode ocasionar até o abandono precoce da prática esportiva ${ }^{3}$, pois as rotinas estressantes de treinamentos e competições aliadas a sensações de medo e ansiedade podem levar à perda de controle das emoções ${ }^{4}$. A falta de controle destas emoções ao longo do tempo pode desencadear a síndrome de burnout ${ }^{5}$. Neste contexto específico, o burnout tem como principais características a exaustão física e emocional, o reduzido senso de realização esportiva e a desvalorização esportiva ${ }^{6}$.

O burnout tem sido identificado em atletas de diferentes modalidades no contexto internacional7,8, mas na realidade brasileira as pesquisas ainda são incipientes ${ }^{1}$. Dois exemplos recentes são os estudos de Casagrande et al. ${ }^{9}$ e Sobral et al. ${ }^{10}$, que investigaram a síndrome de burnout em atletas brasileiros. Vieira et al. ${ }^{11}$ constataram que o ranking dos atletas brasileiros de voleibol de praia influencia em diferentes dimensões da síndrome de burnout.

Por ter sua origem no estresse crônico, a manifestação de burnout pode ser controlada a partir do processo cognitivo de reconhecimento, avaliação e enfrentamento, conhecido como coping. O uso do coping orientado à tarefa desenvolve um senso de determinação e de desenvolvimento dos recursos internos que podem proteger os atletas contra o desenvolvimento de sintomas de burnout ${ }^{7}$. De outra forma, o uso do coping permite enfrentar as demandas e diminuir a suscetibilidade aos efeitos da síndrome ${ }^{8}$. Consequentemente, pesquisadores buscaram identificar e analisar as estratégias de coping utilizadas pelos atletas em diferentes contextos socioculturais e esportivos ${ }^{12,13}$. Por exemplo, Coimbra et al. ${ }^{14}$ analisaram as estratégias de coping de 375 atletas brasileiros de ambos os sexos pertencentes a diversas modalidades esportivas e níveis técnicos, e constataram que as habilidades de coping são dependentes do contexto.

Em vista disso, o monitoramento da síndrome de burnout e das estratégias de coping sob uma perspectiva longitudinal tem sido recomendado nas ciências do esporte ${ }^{15}$. O delineamento longitudinal, por acompanhar os atletas durante uma longa temporada, pode facilitar o entendimento das relações entre a síndrome de burnout e as estratégias de coping. Apesar destas características, não foram encontrados estudos longitudinais que relacionem as dimensões da síndrome de burnout e as estratégias de coping no contexto esportivo.

Vieira et al. ${ }^{11}$ avaliaram transversalmente atletas brasileiros de voleibol de praia e Schellenberg et al. ${ }^{16}$ analisaram atletas universitários de voleibol em uma curta temporada de três meses. Os estudos mostraram a interferência do coping na percepção de burnout ${ }^{16}$, bem como a correlação inversa entre o emprego das estratégias de coping e os indicadores de burnout ${ }^{11}$.

Dessa forma, o presente estudo tem como objetivo analisar a percepção das dimensões de burnout e as estratégias de coping mediante delineamento-longitudinal durante quatro momentos de uma temporada esportiva, e correlacionar as características temporais de burnout e coping em atletas brasileiros de voleibol masculino. A primeira hipótese do estudo é que os níveis de burnout serão mais elevados nas competições de maior importância, ou seja, ao final da temporada. A segunda hipótese é que o uso de maior quantidade de estratégias de coping deverá apresentar correlação inversa com a percepção de burnout.

\section{MÉTODOS}

Participaram do estudo 15 atletas de voleibol do sexo masculino, todos pertencentes a uma única equipe do estado de Minas Gerais que disputava a Superliga Nacional, principal competição da modalidade no Brasil. No início do estudo, os participantes apresentavam idade média de 24,00 \pm 3,55 anos, participavam de competições oficiais há $9,40 \pm 3,11$ anos e iniciaram a prática do voleibol com idade média de 13,27 $\pm 2,63$ anos. Os procedimentos do estudo foram aprovados pelo Comitê de Ética em Pesquisa da Universidade Federal de Minas Gerais (CAAE-0362.0.203.000-11). Todos os participantes assinaram o Termo de Consentimento Livre e Esclarecido.

\section{Instrumentos}

Foi utilizada a versão traduzida do Athlete Burnout Questionnaire $(A B Q)^{17}$, questionário composto por 15 itens que mensuram a frequência de sentimentos relativos às três dimensões do burnout em atletas: exaustão física e emocional; reduzido senso de realização esportiva e desvalorização da modalidade esportiva ${ }^{18}$. As respostas são apresentadas em uma escala do tipo Likert que varia de "quase nunca" (1) a "quase sempre" (5).

O ABQ para uso no Brasil apresenta consistência interna aceitável e validade de construto ${ }^{17}$. Consequentemente, tem sido utilizado com frequência no contexto esportivo brasileiro 9,10,11.

Também foi utilizado o Athletic Coping Skills Inventory (ACSI-28) ${ }^{19} \mathrm{em}$ sua versão adaptada para o Português ${ }^{14}$. O questionário é composto por 28 itens que se referem a um dos sete fatores que representam estratégias de coping no esporte: lidar com adversidades; desempenho sob pressão; metas/preparação mental; concentração; livre de preocupação; confiança/motivação; e treinabilidade. As respostas são apresentadas em uma escala do tipo Likert que varia de "quase nunca" (0) a "quase sempre" (3). Especificamente para a versão brasileira, três itens associados às dimensões confiança/motivação, concentração e treinabilidade foram removidos por não apresentarem cargas fatoriais suficientes. 


\section{Procedimentos}

A coleta de dados foi realizada em quatro momentos: fase de preparação ou pré-temporada (M1-mês de julho), Campeonato Estadual (M2-mês de setembro), primeiro turno da Superliga, principal competição do país (M3-mês de dezembro), e durante o segundo turno da Superliga (M4-mês de fevereiro), momento decisivo para a definição dos classificados à próxima fase (playoffs).

\section{Análise estatística}

A verificação da normalidade da distribuição dos dados foi realizada através do uso do teste de Shapiro-Wilk. O teste apontou uma distribuição de não normalidade dos dados, o que determinou o emprego de testes não-paramétricos. Dessa forma, o comportamento das varáveis referentes ao burnout e das estratégias de coping ao longo da temporada foi verificado através do teste de Friedman, com post hoc de Wilcoxon.

O coeficiente de correlação de Spearman (rho) foi empregado para verificar associações entre os indicadores das dimensões de burnout e as estratégias de coping em cada momento da temporada. Foram considerados a significância estatística e um valor mínimo crítico de rho > 0,40 como aceitável para as análises, pois corresponde ao limite inferior da intensidade moderada para a correlação entre variáveis ${ }^{20}$. Os procedimentos estatísticos foram calculados pelo pacote estatístico Prisma, versão 6 (GraphPad Software Inc., San Diego, CA, EUA). O nível de significância adotado foi de $p<0,05$.

\section{RESULTADOS}

A Tabela 1 apresenta os dados descritivos para os indicadores de burnout. A análise da dimensão exaustão física e emocional não mostrou aumento significante da percepção do burnout nos quatro momentos avaliados ( $\left.X^{2}=3,48 ; p=0,32\right)$. Também não houve aumento significante da dimensão reduzido senso de realização esportiva $\left(X^{2}=6,20 ; p=0,10\right)$. Contudo, houve aumento significativo da dimensão desvalorização esportiva entre os quatro momentos avaliados $\left(X^{2}=8,48 ; p=0,04\right)$. O post hoc detectou que os aumentos ocorreram do $M 2$ para o $M 4(p=0,01)$. Por fim, os dados do índice global de burnout também aumentaram ao longo dos quatro momentos avaliados, $\left(X^{2}=8,78 ; p=0,03\right)$. 0 post hoc detectou que o índice global de burnout aumentou de $M 2$ para $M 4(p<0,01)$.
As Tabelas 2 e 3 apresentam os dados descritivos para os indicadores de coping. $O$ teste de Friedman não mostrou diferenças significantes na comparação entre os quatro momentos para todas as estratégias de coping: lidar com adversidades $\left(X^{2}=0,65 ; p=0,89\right)$, desempenho sob pressão $\left(X^{2}=0,44 ; p=0,93\right)$, metas/preparação mental $\left(X^{2}=1,70 ; p=0,64\right)$, livre de preocupação $\left(X^{2}=4,30 ; p=0,23\right)$, concentração $\left(X^{2}=3,02 ; p=0,39\right)$, confiança/motivação $\left(X^{2}=0,87 ; p=0,83\right)$ e treinabilidade $\left(X^{2}=4,86\right.$; $p=0,18)$. Também não foram observadas diferenças significativas para os recursos pessoais de coping $\left(X^{2}=1,18 ; p=0,76\right)$.

A Tabela 4 apresenta as correlações entre os indicadores de burnout e coping. Foram observadas correlações inversas, significativas e moderadas entre a dimensão de burnout reduzido senso de realização esportiva e a estratégia de coping confiança/motivação em todos os momentos da temporada (M1: rho=-0,56, $\mathrm{p}<0,05$; $\mathrm{M} 2$ : rho $=-0,57$, $p<0,05$; M3: rho=-0,53, $p<0,05 ; M 4$ : rho=-0,61, $p<0,05)$. Também houve correlação significativa, inversa e forte entre a dimensão desvalorização esportiva e a estratégia lidar com adversidades ( $r h o=-0,78, p<0,05)$ no momento M2. No mesmo momento houve o mesmo padrão de correlação entre o índice global de burnout e a estratégia concentração ( rho $=-0,78, \mathrm{p}<0,05)$. No momento $\mathrm{M} 3$, a dimensão reduzido senso de realização esportiva e a estratégia metas/preparação mental ( $r h o=-0,53$, $p=0,05)$ apresentaram correlação inversa, significativa e moderada.

\section{DISCUSSÃO}

O presente estudo analisou o burnout e as estratégias de coping ao longo de uma temporada, considerando que o uso de estratégias de coping pode minimizar os sintomas de burnout. Para tanto, realizamos medições ao longo de uma temporada em uma equipe de voleibol masculino que disputava a principal competição do Brasil. A primeira hipótese testada foi se o burnout aumenta nas competições mais importantes e a segunda foi se as estratégias de coping apresentam correlações inversas com os índices de burnout. As duas hipóteses foram confirmadas.

A percepção das dimensões de burnout mostrou indicadores mais elevados de desvalorização esportiva no momento final da temporada (M4), sugerindo que as cobranças por desempenho aliadas ao acúmulo das rotinas competitivas influenciam a falta de interesse, desejo e

Tabela 1. Dados descritivos para as variáveis de burnout $(n=15)$.

\begin{tabular}{|c|c|c|c|c|c|c|c|c|c|c|c|c|c|c|c|c|}
\hline & \multicolumn{4}{|c|}{ EFE } & \multicolumn{4}{|c|}{ RSR } & \multicolumn{4}{|c|}{ DES } & \multicolumn{4}{|c|}{ IGB } \\
\hline & M1 & M2 & M3 & M4 & M1 & M2 & M3 & M4 & M1 & M2 & M3 & M4 & M1 & M2 & M3 & M4 \\
\hline $\mathrm{Md}$ & 1,60 & 1,60 & 2,40 & 2,40 & 1,80 & 1,60 & 2,00 & 2,40 & 1,20 & 1,20 & 1,60 & 2,00 & 1,53 & 1,73 & 2,00 & 2,27 \\
\hline Mín & 1,00 & 1,20 & 1,00 & 1,00 & 1,00 & 1,00 & 1,20 & 1,00 & 1,00 & 1,00 & 1,00 & 1,00 & 1,13 & 1,07 & 1,13 & 1,07 \\
\hline Máx & 2,60 & 2,80 & 4,60 & 4,40 & 3,00 & 2,80 & 2,80 & 3,00 & 2,20 & 2,60 & 3,00 & $3,60^{*}$ & 2,40 & 2,40 & 3,33 & $3,40^{*}$ \\
\hline
\end{tabular}

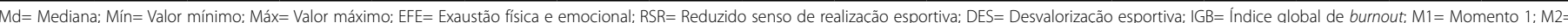
Momento 2; M3= Momento 3; M4= Momento 4. ${ }^{*} \mathrm{p}<0,05$ (em relação a M2).

Tabela 2. Dados descritivos para as estratégias de coping $(n=15)$.

\begin{tabular}{|c|c|c|c|c|c|c|c|c|c|c|c|c|c|c|c|c|}
\hline & \multicolumn{4}{|c|}{ LA } & \multicolumn{4}{|c|}{ DP } & \multicolumn{4}{|c|}{ PM } & \multicolumn{4}{|c|}{ LP } \\
\hline & M1 & M2 & M3 & M4 & M1 & M2 & M3 & M4 & M1 & M2 & M3 & M4 & M1 & M2 & M3 & M4 \\
\hline Md & 7,00 & 8,00 & 7,00 & 8,00 & 9,00 & 8,00 & 9,00 & 8,00 & 10,00 & 8,00 & 9,00 & 8,00 & 7,00 & 5,00 & 5,00 & 5,00 \\
\hline Mín & 4,00 & 4,00 & 5,00 & 3,00 & 5,00 & 4,00 & 6,00 & 6,00 & 4,00 & 4,00 & 5,00 & 4,00 & 0,00 & 0,00 & 3,00 & 0,00 \\
\hline Máx & 11,00 & 12,00 & 12,00 & 12,00 & 12,00 & 12,00 & 12,00 & 12,00 & 12,00 & 12,00 & 12,00 & 12,00 & 10,00 & 9,00 & 9,00 & 10,00 \\
\hline
\end{tabular}

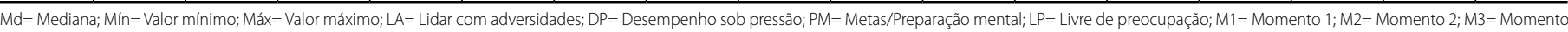
3; M4= Momento 4

Tabela 3. Dados descritivos para as estratégias de coping $(n=15)$. Continuação.

\begin{tabular}{|c|c|c|c|c|c|c|c|c|c|c|c|c|c|c|c|c|}
\hline & \multicolumn{4}{|c|}{$\mathrm{CO}$} & \multicolumn{4}{|c|}{$\mathrm{CM}$} & \multicolumn{4}{|c|}{ TR } & \multicolumn{4}{|c|}{ RPC } \\
\hline & M1 & M2 & M3 & M4 & M1 & M2 & M3 & M4 & M1 & M2 & M3 & M4 & M1 & M2 & M3 & M4 \\
\hline Md & 6,00 & 6,00 & 5,00 & 5,00 & 7,00 & 7,00 & 7,00 & 7,00 & 8,00 & 6,00 & 6,00 & 6,00 & 54,00 & 52,00 & 51,00 & 50,00 \\
\hline Mín & 3,00 & 3,00 & 4,00 & 4,00 & 3,00 & 4,00 & 5,00 & 4,00 & 4,00 & 4,00 & 5,00 & 4,00 & 33,00 & 30,00 & 37,00 & 36,00 \\
\hline Máx & 9,00 & 9,00 & 9,00 & 9,00 & 9,00 & 9,00 & 9,00 & 9,00 & 9,00 & 9,00 & 9,00 & 9,00 & 64,00 & 61,00 & 66,00 & 62,00 \\
\hline
\end{tabular}


Tabela 4. Correlações entre burnout e coping $(n=15)$

\begin{tabular}{|c|c|c|c|c|c|c|c|c|}
\hline \multicolumn{9}{|c|}{ M1 (Pré-Temporada) } \\
\hline & LA & DP & PM & $\mathrm{CO}$ & LP & CM & TR & RPC \\
\hline EFE & 0,07 & 0,17 & 0,26 & 0,19 & $-0,51$ & 0,20 & $-0,31$ & 0,01 \\
\hline RSR & $-0,17$ & $-0,47$ & $-0,36$ & $-0,12$ & $-0,64^{*}$ & $-0,56^{*}$ & $-0,29$ & $-0,66^{*}$ \\
\hline DES & $-0,11$ & $-0,42$ & 0,08 & 0,11 & 0,01 & $-0,09$ & $-0,43$ & $-0,21$ \\
\hline IGB & $-0,03$ & $-0,30$ & $-0,01$ & 0,09 & $-0,60^{*}$ & $-0,20$ & $-0,42$ & $-0,39$ \\
\hline \multicolumn{9}{|c|}{ M2 (Campeonato Estadual) } \\
\hline & LA & DP & PM & $\mathrm{CO}$ & LP & $\mathrm{CM}$ & TR & RPC \\
\hline EFE & $-0,18$ & $-0,02$ & $-0,15$ & $-0,42$ & $-0,04$ & $-0,01$ & $-0,18$ & $-0,24$ \\
\hline RSR & $-0,68^{*}$ & $-0,59^{*}$ & $-0,38$ & $-0,70^{*}$ & 0,02 & $-0,57^{*}$ & 0,06 & $-0,52$ \\
\hline DES & $-0,78^{*}$ & $-0,52^{*}$ & $-0,43$ & $-0,64^{*}$ & 0,21 & $-0,22$ & $-0,07$ & $-0,51$ \\
\hline IGB & $-0,67^{*}$ & $-0,40$ & $-0,47$ & $-0,78^{*}$ & $-0,01$ & $-0,17$ & $-0,36$ & $-0,58^{*}$ \\
\hline \multicolumn{9}{|c|}{ M3 (Primeiro Turno da Superliga) } \\
\hline & LA & DP & PM & CO & LP & CM & TR & RPC \\
\hline EFE & 0,15 & 0,04 & 0,20 & 0,41 & $-0,66^{*}$ & 0,23 & $-0,29$ & $-0,05$ \\
\hline RSR & $-0,27$ & $-0,51$ & $-0,53^{*}$ & $-0,15$ & $-0,14$ & $-0,53^{*}$ & $-0,04$ & $-0,45$ \\
\hline DES & $-0,29$ & $-0,42$ & $-0,42$ & $-0,22$ & $-0,23$ & $-0,14$ & $-0,27$ & $-0,50$ \\
\hline IGB & $-0,04$ & $-0,25$ & $-0,16$ & 0,10 & $-0,55^{*}$ & 0,00 & $-0,36$ & $-0,34$ \\
\hline \multicolumn{9}{|c|}{ M4 (Segundo Turno da Superliga) } \\
\hline & LA & DP & PM & $\mathrm{CO}$ & LP & $\mathrm{CM}$ & TR & RPC \\
\hline EFE & $-0,04$ & $-0,01$ & $-0,30$ & 0,24 & $-0,63^{*}$ & $-0,47$ & 0,07 & $-0,36$ \\
\hline RSR & $-0,39$ & $-0,10$ & $-0,37$ & $-0,16$ & $-0,36$ & $-0,61^{*}$ & $-0,19$ & $-0,50$ \\
\hline DES & $-0,01$ & $-0,24$ & $-0,36$ & $-0,09$ & $-0,47$ & $-0,39$ & $-0,09$ & $-0,43$ \\
\hline IGB & $-0,13$ & $-0,06$ & $-0,41$ & 0,11 & $-0,67^{*}$ & $-0,57^{*}$ & $-0,01$ & $-0,50$ \\
\hline
\end{tabular}

$\mathrm{LA}=$ Lidar com adversidades; $\mathrm{DP}=$ Desempenho sob pressão; $\mathrm{PM}=$ Metas/Preparação mental; $\mathrm{CO}=$ Concentração; $L P=$ Livre de preocupaçăo; $C M=$ Confiança/Motivação; $T R=$ Treinabilidade; $R P C=$ Recursos pessoais de coping; $\mathrm{EFE}=$ Exaustão física e emocional; $\mathrm{RSR}=$ Reduzido senso de realização esportiva; $\mathrm{DES}=$ Desvalorização esportiva; IGB = Índice global de burnout. ${ }^{*} \mathrm{p}<0,05$

preocupação em relação à carreira atlética ${ }^{21}$, ou seja, os atletas perceberam o segundo turno da Superliga (M4) como mais importante que o Campeonato Estadual (M2), reforçando a compreensão do burnout como um processo que se desenvolve em resposta ao estresse crônico resultante das intensas rotinas de treinamentos, viagens e competições ${ }^{22}$. Lai e Wiggins ${ }^{23}$ também encontraram aumento no índice global de burnout em atletas de futebol universitário ao longo de cinco meses. $\mathrm{O}$ reduzido senso de realização esportiva durante as competições também aumentou em atletas de rúgbi ${ }^{24}$.

Outra explicação para o aumento do burnout é que no $\mathrm{M} 2$ a equipe disputava com relativo sucesso o Campeonato Estadual, chegando à semifinal da competição com importantes vitórias. Ao contrário, o M4 representou o segundo turno da competição principal e a equipe encontrava-se nas últimas colocações. Os piores resultados parecem refletir diretamente nas variáveis do burnout.

Esperava-se que os escores do índice global de burnout e de suas dimensões aumentassem ao longo da temporada devido às demandas situacionais, bem como pela percepção do ambiente da equipe $^{24}$. Nossos resultados mostraram aumento dos indicadores de burnout no M4, a competição mais importante da temporada. Nesta competição existem diversas viagens, jogos duas vezes na semana, cobranças dos patrocinadores por resultados positivos que classifiquem a equipe para os playoffs, além dos resultados esportivos per si. No conjunto, estas demandas situacionais podem ter influenciado a percepção dos atletas. Tais resultados dão suporte ao modelo cognitivo-afetivo de burnout proposto por Smith ${ }^{22}$. Neste modelo, as demandas situacionais constituem o primeiro estágio de desenvolvimento da síndrome e são seguidas pela avaliação cognitiva, que terá como consequências as respostas fisiológicas (e.g., insônia) e comportamentais (e.g., estratégias de coping).
Em relação às estratégias e aos recursos pessoais de coping, não foram encontradas diferenças significativas entre os quatro momentos da temporada. Esses resultados indicam um perfil estável no emprego das estratégias de coping ao longo do tempo e das situações. Talvez cada atleta utilize estratégias preferidas de coping, já que Louvet et al. ${ }^{25}$ reforçaram a caracterização do coping como um traço de personalidade ao identificar estabilidade nas estratégias empregadas por atletas ao longo de três competições.

A revisão sistemática sobre coping no esporte de Nicholls e Polman ${ }^{12}$ apresentou resultados que dão suporte à abordagem de traço, outros à abordagem de estado e outros à coexistência das duas abordagens. Ainda não estão claras as razões pelas quais os atletas apresentam consistência (abordagem de traço) ou variabilidade (abordagem de estado) em suas estratégias de coping ${ }^{26}$. Os resultados deste estudo dão alguns indícios à predominância da abordagem de traço. Talvez seja interessante que estudos futuros busquem compreender os motivos de adoção das estratégias de coping. Com base nestes dados, poder-se-á delinear o treinamento para otimizar o controle de situações estressantes. Apesar das estratégias de coping se manterem estáveis, o burnout aumentou, o que denota a necessidade de ajustes ao longo da temporada.

A análise das correlações entre burnout e coping mostrou que no primeiro período competitivo (M2) a dimensão desvalorização esportiva teve correlação significativa, forte e inversa à estratégia lidar com adversidades. A estratégia de lidar com adversidades se mostrou importante para os atletas valorizarem a vivência esportiva, fator relevante para manter o envolvimento na prática esportiva. Ainda em M2, o índice global de burnout teve correlação significativa, forte e inversa à estratégia concentração, evidenciando associação entre a falta de concentração e a percepção da síndrome ${ }^{27}$, especialmente quando a equipe alcançou a semifinal do Campeonato Estadual.

No M3, houve correlação inversa entre a dimensão reduzido senso de realização esportiva e a estratégia metas/preparação mental, contrário ao encontrado por Vieira et al. ${ }^{11}$. Contudo, os resultados do presente estudo mostram ser importante estabelecer metas realistas ${ }^{2}$ e desenvolver força mental ${ }^{28}$ para o controle dos indicadores de burnout durante as competições de maior importância.

No M4 foram identificadas correlações inversas, significativas e moderadas da dimensão reduzido senso de realização esportiva e do índice global de burnout com a estratégia confiança/motivação. As mesmas correlações aconteceram entre reduzido senso de realização esportiva e confiança/motivação nos momentos M1, M2 e M3, oposto aos achados de Vieira et al. ${ }^{11}$. Contudo, os dados foram coletados em um momento único durante uma etapa do Circuito Brasileiro de voleibol de praia. O presente estudo permitiu a observação das variáveis em momentos distintos da temporada, o que favorece a compreensão das alterações nas duas variáveis (burnout e coping), o que não encontramos na literatura.

Os achados do presente estudo corroboram as evidências de que a elevada motivação intrínseca tem relação inversa ao burnout, ou seja, atletas com altos níveis de motivação intrínseca se percebem como competentes e autônomos, tornando-se menos suscetíveis à manifestação da síndrome. Cresswell e Eklund ${ }^{29}$ já mostraram associação entre o surgimento do burnout a partir de comprometimento na motivação do atleta, e Raedeke ${ }^{18}$ observou que a motivação se constituiu em um tema inerente à experiência de burnout. Portanto, a motivação é uma estratégia de coping que interfere na percepção de burnout em atletas de alto rendimento.

Este estudo utilizou instrumentos psicométricos para dimensionar burnout e coping, que são recorrentemente utilizados. Contudo, entrevistas 
qualitativas poderiam fornecer informações subjetivas sobre as experiências individuais de burnout e coping, bem como de suas interações. Sua utilização em estudos futuros pode oferecer informações complementares sobre os fenômenos investigados.

Estudos encontrados relatam um corte transversal, e de modo genérico, como são percebidas as dimensões de burnout e utilizadas as estratégias de coping ao longo de uma temporada ${ }^{30}$. Esse estudo longitudinal proporciona o retrato dos diferentes padrões de mudança e estabilidade da síndrome de burnout e das estratégias de coping em atletas de alto rendimento, dando um passo adiante no entendimento da relação entre burnout e coping.

\section{CONCLUSÃO}

Os achados evidenciaram percepção mais elevada relacionada ao burnout em atletas a partir do acúmulo de treinamentos e competições, bem como da exigência por ótimo desempenho na competição-chave. Algumas estratégias de coping, como confiar em suas capacidades e estar motivado para o bom desempenho, podem ser úteis no processo de prevenção e controle dos indicadores de burnout.

Todos os autores declararam não haver qualquer potencial conflito de interesses referente a este artigo.

CONTRIBUIÇÕES DOS AUTORES: Cada autor contribuiu individual e significativamente para o desenvolvimento do manuscrito. DAP (0000-0003-2163-5606)* e HU (0000-0003-0317-1940)* foram os principais contribuintes na redação do manuscrito. DAP, MGBF (0000-0003-1219-8379)* e DRC (0000-0002-6055-0667)* acompanharam os atletas e reuniram dados ao longo da temporada esportiva. DAP e HU avaliaram os dados da análise estatística. DAP e PBD (0000-0003-4969-4544)* realizaram a pesquisa bibliográfica. DAP, HU e MGBF contribuíram com o conceito intelectual do estudo. Todos os autores participaram ativamente da discussão dos resultados e realizaram a revisão da versão final do manuscrito. *Número ORCID (Open Researcher and Contributor ID).

\section{REFERÊNCIAS}

1. Pires DA, Santiago ML, Samulski DM, Costa VT. A Síndrome de burnout no esporte brasileiro. Rev Educ Fís/UEM. 2012;23(1):131-39.

2. Weinberg RS, Gould D. Fundamentos da Psicologia do Esporte e do Exercício. 4a. ed. Porto Alegre: Artmed; 2008.

3. Isoard-Gautheur S, Guillet-Descas E, Gustafsson $\mathrm{H}$. Athlete burnout and the risk of dropout among young elite handball players. Sport Psychol. 2016;30(2):123-30.

4. Bara Filho MG, Garcia FG. Motivos do abandono no esporte competitivo: um estudo retrospectivo. Rev Bras Educ Fís Esp. 2008;22(4):293-300.

5. Pires DA, Brandão MR, Machado AA. A Síndrome de burnout no esporte. Motriz. 2005;11(3):147-53.

6. Raedeke, TD, Smith, AL. Development and preliminary validation of an athlete burnout measure. $J$ Sport Exerc Psychol. 2001;23:238-306

7. Goodger K, Gorely T, Lavallee D, Harwood C. Burnout in sport: a systematic review. Sport Psychol. 2007;21(2):127-51

8. Hill AP, Hall HK, Appleton PR. Perfectionism and athlete burnout in junior elite athletes: the mediating role of coping tendencies. Anxiety Stress Coping. 2010;23(4):415-30

9. Casagrande PO, Andrade A, Viana MS, Vasconcellos DI. Burnout em tenistas brasileiros infanto-juvenis. Motricidade. 2014;10(2):60-71

10. Sobral AS, Oliveira L, Oliveira LS, Santos RM, Brito AF. Associação entre as cargas de treino impostas a jogadores amadores de rugby sevens e a síndrome de burnout. Motricidade. 2014;10(2):25-35

11. Vieira LF, Caruzzo NM, Aizava PV, Rigoni, PA. Análise da síndrome de "burnout" e das estratégias de "coping" em atletas brasileiros de vôlei de praia. Rev Bras Educ Fís Esp. 2013;27(2):269-76.

12. Nicholls AR, Polman RC. Coping in sport: a systematic review. J Sports Sci. 2007;25(1):11-31.

13. Reeves C, Nicholls A, Mckenna J. The effects of a coping intervention on coping self-efficacy, coping effectiveness, and subjective performance among adolescent soccer players. Int J Sport Exerc Psychol. 2011;9(2):126-42.

14. Coimbra DR, Bara Filho MG, Andrade A, Miranda R. Habilidades psicológicas de coping em atletas brasileiros. Motricidade. 2013;9(1):94-105

15. Lemyre PN, Hall HK, Roberts GC. A social cognitive approach to burnout in elite athletes. Scand J Med Sci Sports. 2008;18(2):221-34.
16. Schellenberg BJ, Gaudreau P, Crocker PR. Passion and coping: relationships with changes in burnout and goal attainment in collegiate volleyball players. J Sport Exerc Psychol. 2013;35(3):270-80.

17. Pires DA, Brandão MRF, Silva CB. Validação do questionário de burnout para atletas. Rev Educ Fís/ UEM. 2006:17(1):27-36

18. Raedeke TD. Is athlete burnout more than just stress? A sport commitment perspective. J Sport Exerc Psychol. 1997;19: 396-417.

19. Smith RE, Schutz RW, Smoll FL, Ptacek J. Development and Validation of a multidimensional measure of sport-specific psychological skills: the athletic coping skills inventory-28. J Sport Exerc Psychol. 1995;17(4):379-98.

20. Dancey CP, Reidy J. Estatística sem matemática para a psicologia. 5a. ed. Porto Alegre: Penso; 2013

21. Verardi CE, Santos AB, Nagamine KK, Carvalho T, Miyazaki MC. Burnout e enfrentamento em jogadores de futebol: fases pré e durante competição. Rev Bras Med Esporte. 2014;20(4):272-75.

22. Smith R. Toward a cognitive-affective model of athletic burnout. J Sport Psychol. 1986;8(1):36-50.

23. Lai C, Wiggins MS. Burnout perceptions over time in NCAA Division I Soccer Players. Int Sports J. 2003;7(2):120-7.

24. Cresswell SL, Eklund RC. Changes in athlete burnout over a thirty-week "rugby year". J Sci Med Sport. 2006;9(1-2):125-34.

25. Louvet B, Gaudreau P, Menaut A, Genty J, Deneuve P. Longitudinal patterns of stability and change in coping across three competitions: a latent class growth analysis. J Sport Exerc Psychol. 2007;29(1):100-17.

26. McDonough MH, Hadd V, Crocker PR, Holt NL, Tamminen KA, Schonert-Reichl K. Stress and Coping Among Adolescents Across a Competitive Swim Season. Sport Psychol. 2013;27(2):143-55.

27. DiFiori JP, Benjamin HJ, Brenner J, Gregory A, Jayanthi N, Landry GL, et al. Overuse injuries and burnout in youth sports: a position statement from the American Medical Society for Sports Medicine. Clin J Sport Med. 2014;24(1):3-20

28. Gucciardi DF, Gordon S. Development and preliminary validation of the Cricket Mental Toughnes Inventory (CMTI). J Sports Sci. 2009;27(12):1293-310.

29. Cresswell SL, Eklund RC. Motivation and burnout in professional rugby players. Res Q Exerc Sport. 2005;76(3):370-6

30. Todd M, Tennen H, Carney MA, Armeli S, Affleck G. Do we know how we cope? Relating daily coping reports to global and time-limited retrospective assessments. J Pers Soc Psychol. 2004;86(2):310-9. 